\title{
A novel ball joint wear sensor for low-cost structural health monitoring of off-highway vehicles
}

\author{
D. Castagnetti ${ }^{1, a}$, A. Bertacchini ${ }^{1}$, A. Spaggiari ${ }^{1}$, A. $\operatorname{Lesnjanin}^{1}$, L. Larcher $^{1}$, \\ E. Dragoni ${ }^{1}$ And M. Arduini ${ }^{2}$ \\ 1 Departement of Sciences and Methods for Engineering, University of Modena and Reggio Emilia, Via G. Amendola, \\ 2, Campus S. Lazzaro, Pad. Morselli, 42122 Reggio Emilia, Italy \\ 2 Ognibene S.p.A., Via Ing. Enzo Ferrari, 2, 42100 Reggio Emilia, Italy
}

Received 5 December 2014, Accepted 28 March 2015

\begin{abstract}
Mechatronic agricultural machines and equipment are continuously increasing their complexity and cost. In order to ensure their efficiency and reliability and preserve their value, it is important to actively monitor damaging and wear occurring on critical components. This approach needs the introduction of sensors on the machine, which allow continuous monitoring of the residual life of components. This work presents the development and testing of a wear sensor for a ball joint which can be applied for monitoring and diagnosis in off-highway vehicles, automotive and the industrial fields. Many peculiar features make this sensor innovative and contribute to the advance of the technology in the sector: there are no other active sensors for this specific and safety-critical joint; it has an ultra-low power consumption and can be self-powered through energy harvesting; it implements wireless connectivity; it is simple, small size and low cost. This wear sensor for the ball joint is firstly aimed at monitoring the damage of the ball joint placed between the steering actuator and the wheel spindle, since failure of the joint leads to complete loss of steering action. However, the sensor can be applied to any application involving a safety-critical ball joint (e.g. the front suspension of a vehicle). The present work describes the conceptual design and development of the whole mechatronic sensor, which includes the mechanical joint sensor and the electronic board which manages the system. Validation of the ball joint wear sensor, which was performed on an agricultural tractor through tests on a track, proved the reliability of the proposed solution.
\end{abstract}

Key words: Ball joint / wear sensor / preventive diagnosis / active safety / vehicles / wireless node / ultra-low power consumption

\section{Introduction}

Ball joints are spherical bearings obtained by coupling a ball-ended pin with a spherical socket to allow free rotations about a single point. They are frequently used in off-highway vehicles, where ball joints connect the steering actuator to the wheel spindle. In the automotive field ball joints are used, in addition, to connect the members of the front suspension. Consequently, ball joints are a very critical component, since their failure causes either a complete loss of the steering action or determines a sudden loss of vehicle handling, thus leading to severe accidents. Despite this, no simple and low cost solutions are currently available in order to actively monitor the structural integrity and the residual service life of ball

\footnotetext{
a Corresponding author:

davide.castagnetti@unimore.it
}

joints. Only passive solutions, where the joint is manually checked by an operator, are available, see for example [1].

Wear originated by the relative movement between the ball and the socket is the most critical issue for this component, which can lead to failure also in long life ball joints [1-3], as demonstrated by a number of publications in the literature [4-9]. In order to reduce the friction and prevent wear, actual ball joints contain lubricating grease between the ball and the socket interface. Possible failure of the polyurethane sleeve that seals the ball joint and contains the grease leads to double drawback: the leak of the lubricant and the chance of having dirt and debris contamination of the joint. As a consequence, ball joint degradation occurs due to wear of the ball socket system, up to the complete failure which could cause catastrophic consequences (Fig. 1).

Smart ball joint solutions including wear sensors were patented in the last years [10-14]. However, such solutions 


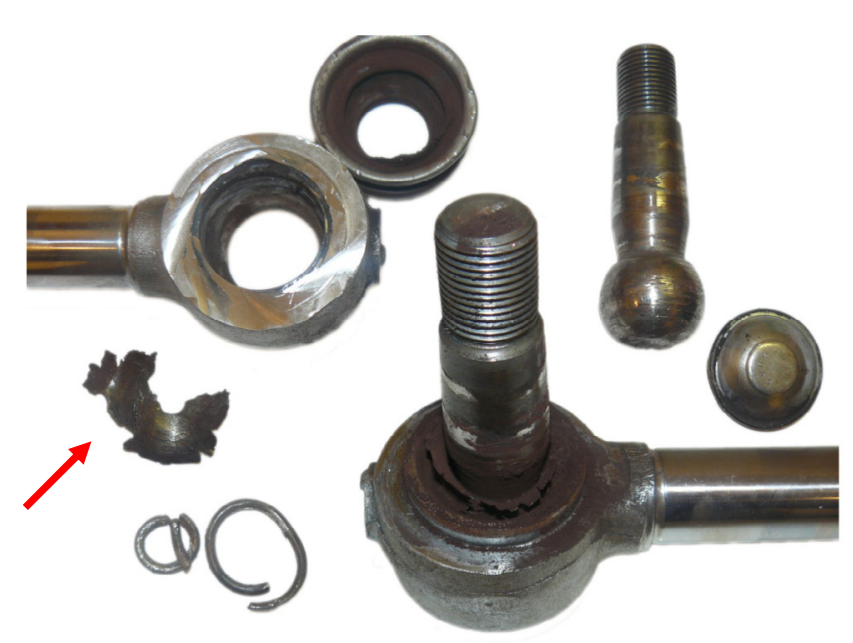

Fig. 1. Ball joint at the end of an endurance test performed without lubricating grease. The arrow highlights the completely failed conical upper bearing.

suffer from some drawbacks: some of them [10-12] need visual inspection, thus preventing the development of automatic methods; other solutions $[13,14]$ monitor wear through an electric circuit whose operation relies on the electric contact between the ball and the socket. The wear of the system components (e.g. ball) originates a gap between the ball and the socket that opens the circuit, largely increasing the electrical resistance. The main limitation of such solution is its considerable energy consumption, which would require a wired connection to power the sensor, practically impossible given the position of the joint.

In this scenario, this paper presents an innovative ball joint wear sensor enabling remote diagnosis, which overcomes the limitations of the existing solutions. The peculiar features of the proposed ball joint wear sensor that make clear how this work contributes to the advance of the technology in the sector are:

I there are no other active sensors on the market for this and safety-critical ball joint;

II the sensor has a ultra-low power consumption and thus can be self-powered through energy harvesting;

III it features wireless connectivity;

IV it is low cost;

$\mathrm{V}$ it has a small size and simple architecture;

VI its simple architecture makes possible an easy integration into existing ball joints.

The wear sensor is able to continuously monitor the wear level of the joint and to measure the joint acceleration. Through wireless connectivity, the sensor periodically sends this information to an electronic central unit (ECU), which collects data from all the wireless sensor nodes on the vehicle. It is worth noting that the information provided by the developed sensor node enhances the overall safety of the vehicle since they monitor the residual service life of the ball joint and thus allow preventive maintenance and diagnosis.

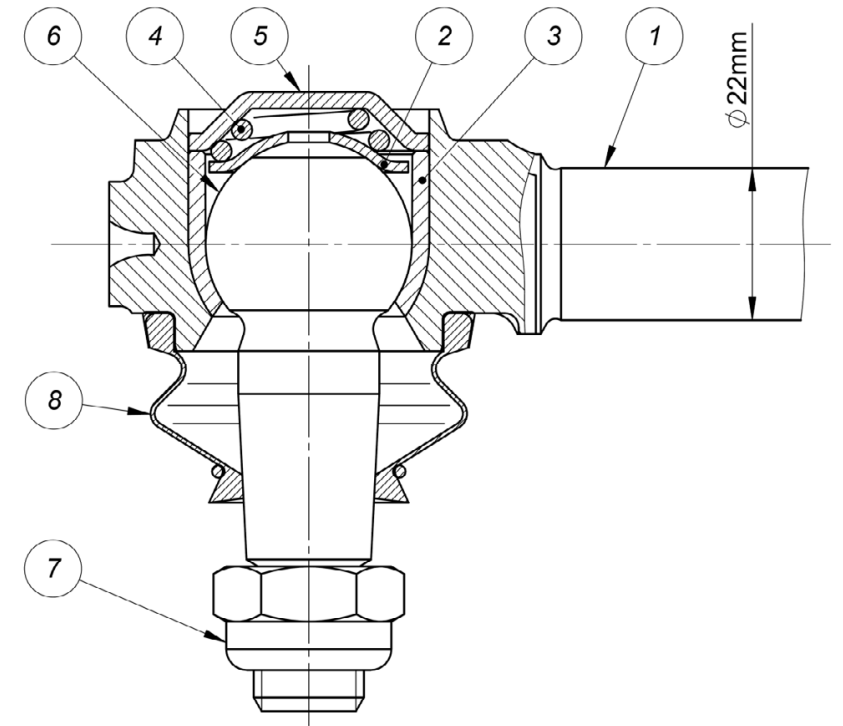

Fig. 2. Sketch of the ball joint: (1) piston rod, (2) upper bearing, (3) lower bearing, (4) helical spring, (5) upper cup, (6) ball-ended pin, (7) plastic-stop nut, (8) polyurethane sleeve.

The design and development of the ball joint wear sensor was performed according to the quality function deployment method [15]. The work involves two steps. The first step involves the conceptual and detailed design phase of both the mechanical system and electronic circuit. The second step develops the prototype and validates it experimentally.

The results show that the proposed ball joint wear sensor represents a valuable solution for structural health monitoring of off-highway vehicles. The same system can be applied to different fields, e.g. automotive or other systems where a ball joint is used, thus contributing to improve the safety.

The work was supported by the ISOTRACTOR project [16], which was motivated by the need to improve safety in tractors and off-highway vehicles since national and international statistics confirm that tractor accident is one of the highest causes of fatality. For example, about 150 fatalities per year occurred in the last years in Italy, while about 100 fatalities per year occurred in the United States [17-22], mainly caused by human errors In this scenario, there is an evident need for on-board control systems capable to actively prevent dangerous operations, such as taking steep paths, thus enhancing machinery operator safety. The ball joint wear sensor was developed in cooperation with Ognibene S.p.A., and tested on an actual steering system mounted on commercial tractors.

\section{System design}

Figure 2 shows the sketch of the ball joint that is installed in steering systems of off-highway vehicles having a power range from $50 \mathrm{Hp}$ to $100 \mathrm{Hp}$. This ball joint connects the hydraulic cylinder piston rod (1) to the wheel spindle. The ball-ended pin (6) is pushed by the lower 
D. Castagnetti et al.: Mechanics \& Industry 16, 507 (2015)

Table 1. Technical specifications of the ball joint wear sensor.

\begin{tabular}{|c|c|c|c|c|c|}
\hline Need No. & Need & Weights & Technical specification & Unit & Value \\
\hline 1 & Low consumption & 5 & Power & $\mu \mathrm{W}$ & $<100$ \\
\hline 2 & Monitors wearing & 5 & Monitors wearing & Binary & Yes/No \\
\hline 3 & Low cost & 5 & Cost & $€$ & $<20$ \\
\hline 4 & Automatic & 3 & Automatic & Binary & Yes/No \\
\hline 5 & $\begin{array}{l}\text { Can be implemented } \\
\text { on different ball joints }\end{array}$ & 3 & $\begin{array}{l}\text { Can be implemented } \\
\text { on different ball joints }\end{array}$ & Binary & Yes/No \\
\hline 6 & Predicts life & 2 & $\begin{array}{l}\text { Predicts life } \\
\text { Error in life prediction }\end{array}$ & $\begin{array}{l}\text { Binary } \\
\text { Weeks }\end{array}$ & $\begin{array}{l}\text { Yes/No } \\
<4\end{array}$ \\
\hline & & & Max vibrations (PSD) & $\mathrm{g}^{2} \cdot \mathrm{Hz}^{-1}$ & 0.2 \\
\hline & & & Average life & Cycles & $>150000$ \\
\hline 7 & Robust & 2 & $\begin{array}{c}\text { IP level } \\
\text { Operating temperature interval } \\
\text { Shock load resistance }\end{array}$ & $\begin{array}{c}{ }^{\mathrm{IP}} \\
{ }^{\circ} \mathrm{C} \\
\text { Binary }\end{array}$ & $\begin{array}{l}>56 \\
-35 \div+90 \\
\text { Yes } / \text { No }\end{array}$ \\
\hline 8 & Uses commercial components & 2 & Uses commercial components & Binary & Yes/No \\
\hline 9 & Small size & 1 & $\begin{array}{l}\text { Volume } \\
\text { Main side length }\end{array}$ & $\begin{array}{c}\mathrm{mm}^{3} \\
\mathrm{~mm}\end{array}$ & $\begin{array}{l}<4000 \\
<30\end{array}$ \\
\hline
\end{tabular}

bearing (3) and the spring (4) against the upper bearing (2). The friction between the ball-ended pin (6) and the upper bearing (2) is reduced by Molykote grease, which is confined by the polyurethane sleeve (8). The use in rough terrain can damage the polyurethane sleeve of the ball joint: the lubricant is washed away and the joint starts to wear. The aim of this work was to design and develop a wear sensor for the ball joint, in order to allow real-time diagnosis and preventive maintenance of the system.

Three main constraints have driven the wear sensor development. First, ultra-low power consumption. Second, a simple integration with the actual ball joint structure. Third, an easy applicability to different ball joints. Table 1 collects all the customer needs and their correlation with quantitative technical specifications.

According to the procedure proposed by Ulrich and Eppinger [15], the customer needs and technical specifications were organized in the house of quality, not reported here for the sake of brevity. The house of quality, together with a functional diagram of the system, has oriented the whole concept generation phase.

This section describes both the design of the ball joint wear sensor and the development of the electronic circuit of the system management.

\subsection{Design of the ball joint wear sensor}

Figure 3 shows the concepts proposed for the ball joint wear sensor inspired by the preliminary investigation of the technical literature [10-14,23-37]. Concepts shown in Figures $3 \mathrm{a}-3 \mathrm{~d}$ rely on monitoring the resistance of an electric connection, which is either very low (i.e. short-circuit, Figs. 3a, 2c, and 2d) or very high (i.e. open Fig. 3b) during normal operation, and it switches to very high and very low value when wear occurs. The circuit is built between the ball pin and the outer case (Fig. 3a), or between the lower bearing and the ball pin (Fig. 3b). By contrast, in Figures $3 \mathrm{c}$ and $2 \mathrm{~d}$ an insert containing different electrical paths is placed in the thickness of the lower bearing or through a hole in the outer case and upper bearing respectively. All these solutions rely on the idea that the wear of the ball joint changes the electrical conductivity of the medium placed in between two conductive elements of the circuit.

The solution illustrated in Figure 3e correlates the wear to the lack of lubricant inside the room of the ball joint, which is detected by measuring the magnetic permeability of the lubricant itself. Another solution is proposed in Figure 3f where the relative displacement occurring during wear between the joint cap and the ball pin is measured using a displacement transducer.

These concepts were qualitatively examined and quantitatively evaluated against the technical specifications reported in Table 1, weighted on their importance. Through a classical screening and scoring matrix [15] (not shown for concision), the concept in Figure 3b was chosen. Since the relative importance of the technical specifications is directly related to the priority of the client needs, which has been defined at the beginning of the project, no sensitivity analysis to these weights was performed.

Figure 4 shows the detailed technical sketch of the concept proposed according to Figure 3b. This solution shows three main advantages compared to those proposed in Figure 3 and compared to the patents traceable in the literature [10-14]. First, the wear sensor relies on an open circuit in normal conditions, hence the power consumption is zero unless the failure occurs. Second, this solution is simple, easy to be integrated into the current architecture of ball joints and thus inexpensive. Third, this solution is more reliable compared to other concepts in Figure 3.

The operating principle of the wear sensor is based on the variation of electrical resistance between the pinball (part 6 in Fig. 4) and the upper bearing (part (2) in Fig. 4). In normal conditions (no damage occurred), the upper bearing (part 2 in Fig. 4) is coated by an insulating shield to achieve a very high resistance, i.e. an open circuit between the bearing and the ball (part 6 in Fig. 4). This conical bearing is pressed against the ball-ended pin 


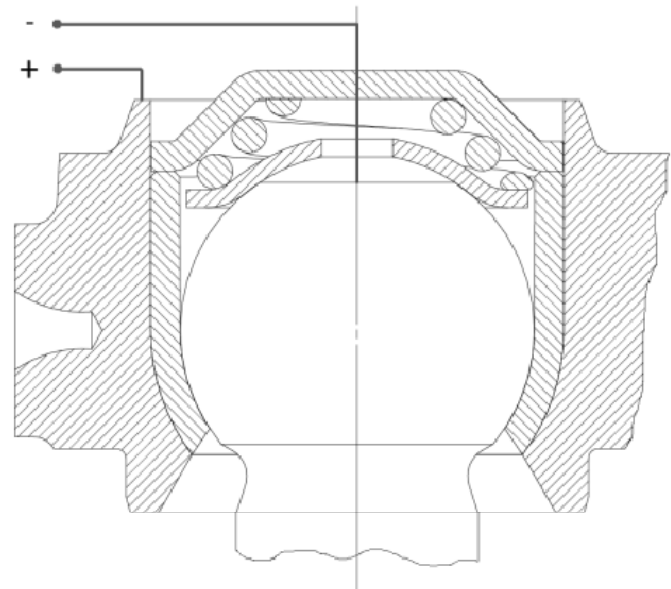

(a)

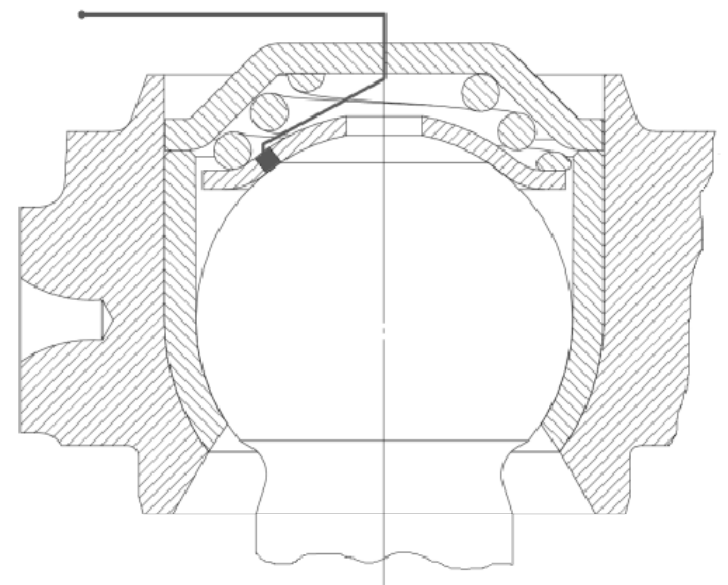

(c)

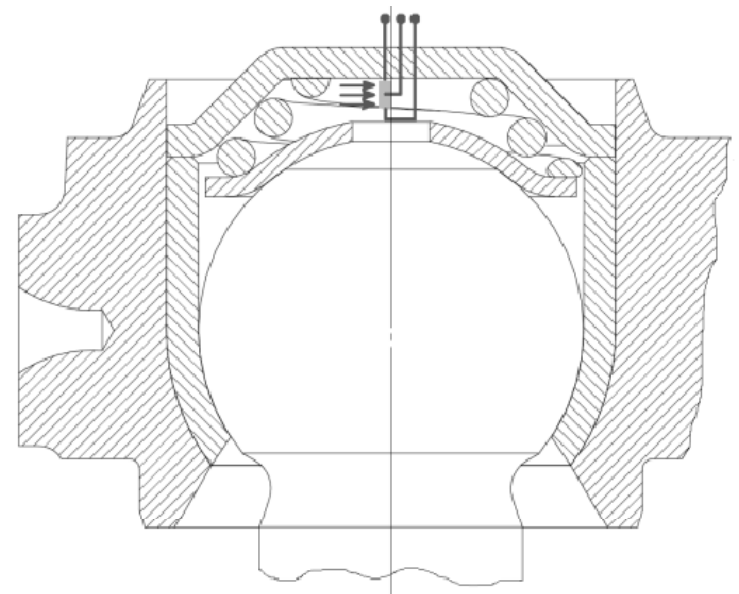

(e)

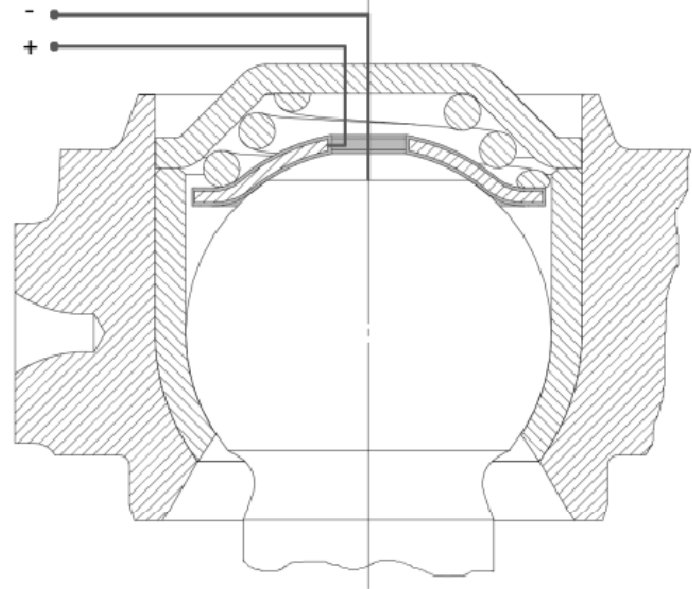

(b)

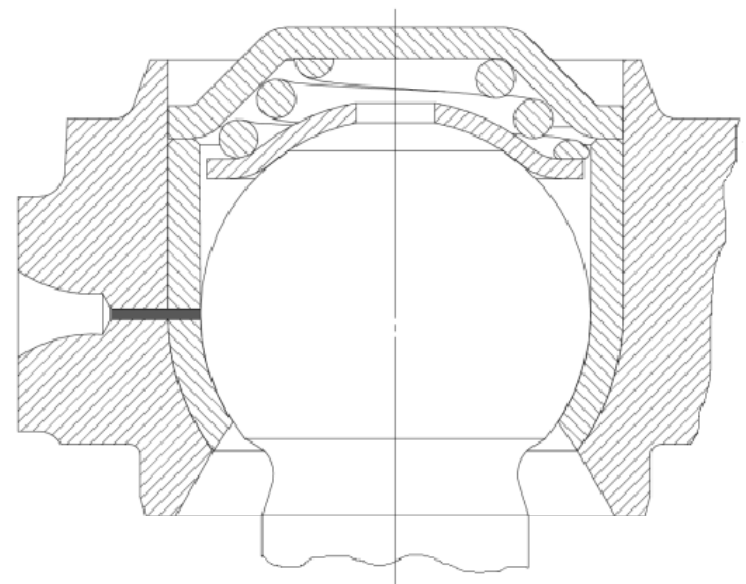

(d)

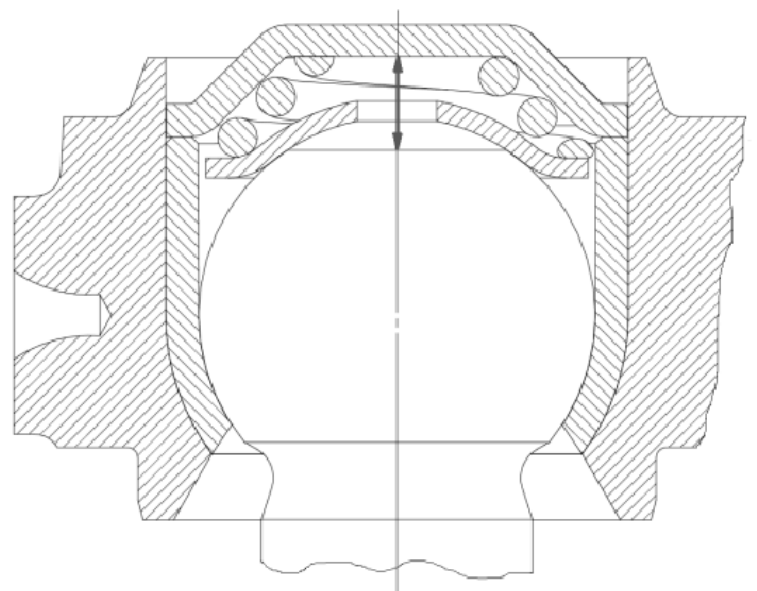

(f)

Fig. 3. Sensor concepts generation: (a) closed circuit between lower bearing and ball-ended pin; (b) open circuit between upper bearing and ball; (c) wearable sensing circuit embedded in the upper bearing, normally closed circuit; (d) wearable sensing circuit embedded in the outer case, normally closed circuit; (e) magnetic permeability sensor to measure the lack of lubricant inside the room of the ball joint; (f) displacement transducer to measure the relative displacement between the ball and the upper cup. 


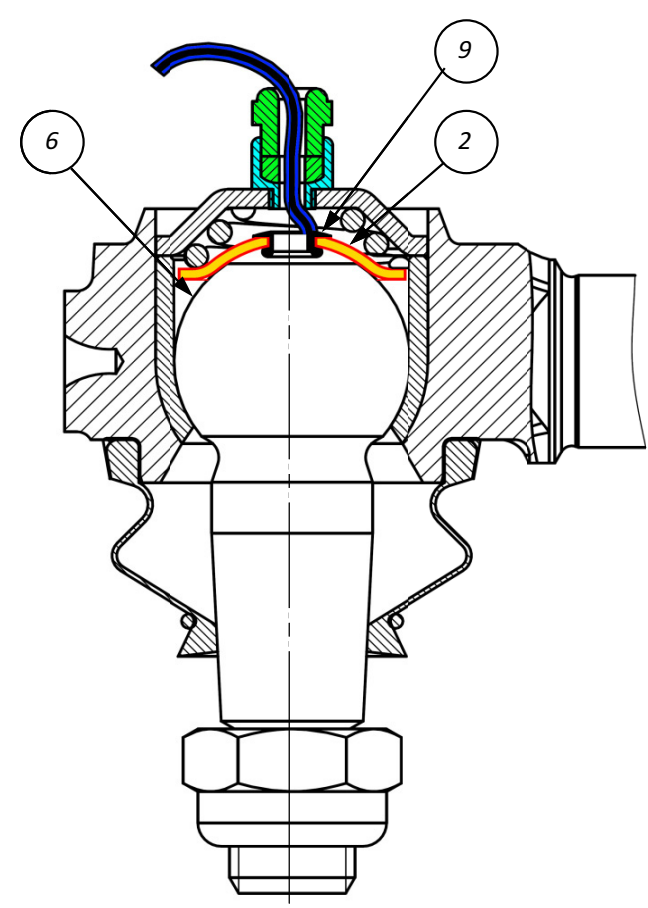

Fig. 4. Section view of the wear sensor included in the ball joint.

by a conical helical spring. In case of a lack of lubricant or in case the system is not correctly sealed thus contaminants get inside the joint, under working loads the wear occurring on the surface of the bearing damages the insulating layer. Through the cable connected to the bearing by means of a rivet (part 9 in Fig. 4) applied in the center hole, it is possible to measure the electric resistance between the bearing and the ball (up to short circuit condition): the lower the electric resistance, the higher the wear in the system. In conclusion, this wear sensor monitors the degradation of the upper bearing (part 2) in the ball joint, which is the most critical element and leads to complete failure of the joint (see Fig. 1).

\subsection{Design of the electronic circuit}

The proposed wireless sensor node allows enhancing the overall safety of the system where the joint is used: the wear status of the joint and the accelerations to which is subjected are delivered to a dedicated ECU which can use these data for preventive maintenance and diagnosis purposes. The system is comprised of two main components: the on-joint sensor (JS) and the on-vehicle control unit, i.e. the Data Manager (DM).

Figure 5 shows the electronic system architecture. In this paper we considered the case study of a joint used in off-highway vehicles: the DM device designed to be positioned on the cabin of the tractor works as the access point for the data transmitted from the JS and other wireless sensors on the vehicle

Thanks to the Control Area Network (CAN) bus connectivity of the DM, the received data can be transmitted

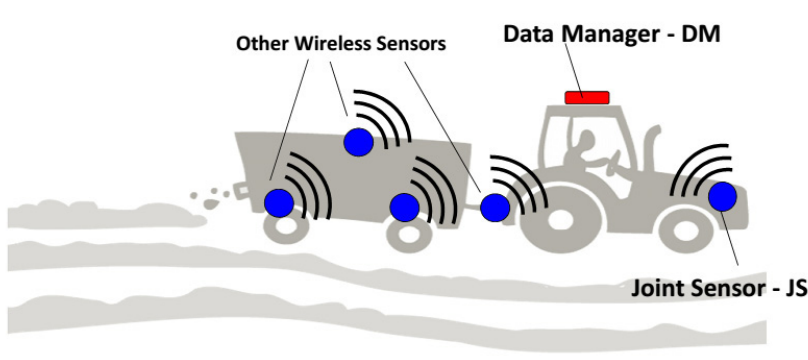

Fig. 5. System architecture.

to the electronic systems onboard and used in combination with other data to monitor the working conditions of the whole vehicle.

The JS is in charge to sense periodically the wear level of the joint and to measure the accelerations occurring during its operation. After each sensing operation, the JS sends the data together with a unique device identifier (i.e. ID) to the DM. In this way the DM can collect data from more than one wireless sensor installed on the vehicle without data conflicts.

\section{Prototype development}

\subsection{Ball joint including wear sensor}

Figure 6 shows the prototype of the ball joint including the wear sensor manufactured according to the technical drawing in Figure 4. The upper cup (part (5) in Fig. 2) and its locking system were slightly modified in order to allow rapid disassembly of the joint, thus simplifying periodic inspections during tests. All other parts of the system belong to a standard ball joint provided by Ognibene S.p.A.. With reference to the sketch shown in Figure 2, the ball-ended pin (6) is made in quenchtempered steel. Two different materials were considered for the upper bearing (2): carburized and hardened mild steel or aluminum. The lower bearing (3) was made with the same carburized and hardened material as the upper bearing. The wires come out from the center of the joint cup through a plastic coupling. The sleeve (8) is made of polyurethane having grade $385 \mathrm{E}$, with hardness equal to $85 \mathrm{ShA}$. All the system is locked by a plastic-stop nut (7). The electrical connection to the upper bearing was obtained by welding a wire to a rivet which is then fixed to the upper bearing (2).

Three different insulating layers were investigated for the upper bearing: (i) a coating made in Rilsan, which is a blend of polyamide 11 and 12 (Tab. 2); (ii) a coating made in Loctite Nordbak 7227 [38], which is a two component, ceramic reinforced epoxy, providing a high gloss, low friction insulating coating (Tab. 3); (iii) an aluminum upper bearing which is coated by a thin electrically nonconductive alumina layer originated through anodic oxidation of the bearing itself. 
Table 2. Physical and mechanical properties of Rilsan.

\begin{tabular}{|c|c|c|c|c|}
\hline $\begin{array}{l}\text { Melting point } \\
\left({ }^{\circ} \mathrm{C}\right) \\
\end{array}$ & $\begin{array}{c}\text { Wear resistance } \\
(\mathrm{ASTM} \text { D4060-14) (mg) }\end{array}$ & $\begin{array}{l}\text { Hardness } \\
\text { (ASTM D2240-05) (Shore D) }\end{array}$ & $\begin{array}{l}\text { Thickness } \\
(\mathrm{mm})\end{array}$ & $\begin{array}{c}\text { Yield strength } \\
(\mathrm{ASTM} \text { D638) (MPa) }\end{array}$ \\
\hline 186 & 15 & 75 & $0.2 \div 0.5$ & 40 \\
\hline
\end{tabular}

Table 3. Physical and mechanical properties of Loctite Nordbak 7227.

\begin{tabular}{cccc}
\hline $\begin{array}{c}\text { Operating temperature } \\
\left({ }^{\circ} \mathrm{C}\right)\end{array}$ & $\begin{array}{c}\text { Compression strength } \\
(\text { ISO } 604)(\mathrm{MPa})\end{array}$ & $\begin{array}{c}\text { Lap shear strength } \\
(\text { ISO } 4587)(\mathrm{MPa})\end{array}$ & $\begin{array}{c}\text { Hardness } \\
(\text { ASTM D2240-05) }\end{array}$ \\
\hline$-30 \div+95$ & 86.2 & 13.8 & 85 \\
\hline
\end{tabular}

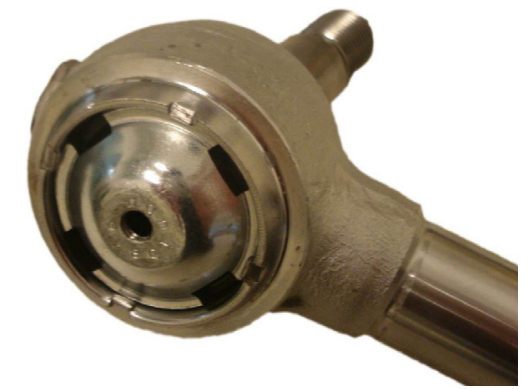

Fig. 6. Prototype of the ball joint including the wear sensor.

\subsection{Electronic system}

\section{Joint sensor}

Figure 7 shows the simplified block diagram of the implemented Joint Sensor (JS) incorporating the wear sensing circuit (bottom left block) described in the former sections. The core of the device is an ultra-low power microcontroller $(\mu \mathrm{C})$ of the MSP430 family from Texas Instruments which achieves good performances with ultralow power consumption (i.e. $\approx 250 \mu \mathrm{A}$ when running at $1 \mathrm{MHz}$, and $\approx 500 \mathrm{nA}$ in sleep mode) and embeds several communication interfaces (i.e. UART, SPI, $\mathrm{I}^{2} \mathrm{C}$ ). The JS is provided with an inertial sensor (LIS3DH, 3-axial MEMS accelerometer from STMicroelectronics) for detecting the motion and measuring joint accelerations. The adopted accelerometer consumes only $10 \mu \mathrm{A}$ with $100 \mathrm{~Hz}$ sampling frequency, while draining only $500 \mathrm{nA}$ in powerdown mode. When in active mode, the sensor can measure acceleration with maximum frequency of $5 \mathrm{kHz}$ and full scale up to $\pm 16 \mathrm{~g}$. The $\mu \mathrm{C}$ periodically polls the accelerometer to detect the presence of vibrations, which allows detecting if the joint is on duty.

In order to further reduce the JS power consumption, a Dynamic Frequency Scaling (DFS) algorithm has been implemented to modulate the clock frequency according to the working condition of the vehicle. In particular, when the vehicle is on duty, the clock frequency rises up to $1 \mathrm{MHz}$ or $8 \mathrm{MHz}$ depending on the particular task (e.g. joint wear sensing or accelerations measurements). By contrast, when the joint is off duty, the clock frequency is reduced to $200 \mathrm{kHz}$.

The wireless communication between JS and DM devices is implemented using the low cost CC2500 lowpower transceiver from Texas Instruments which can be

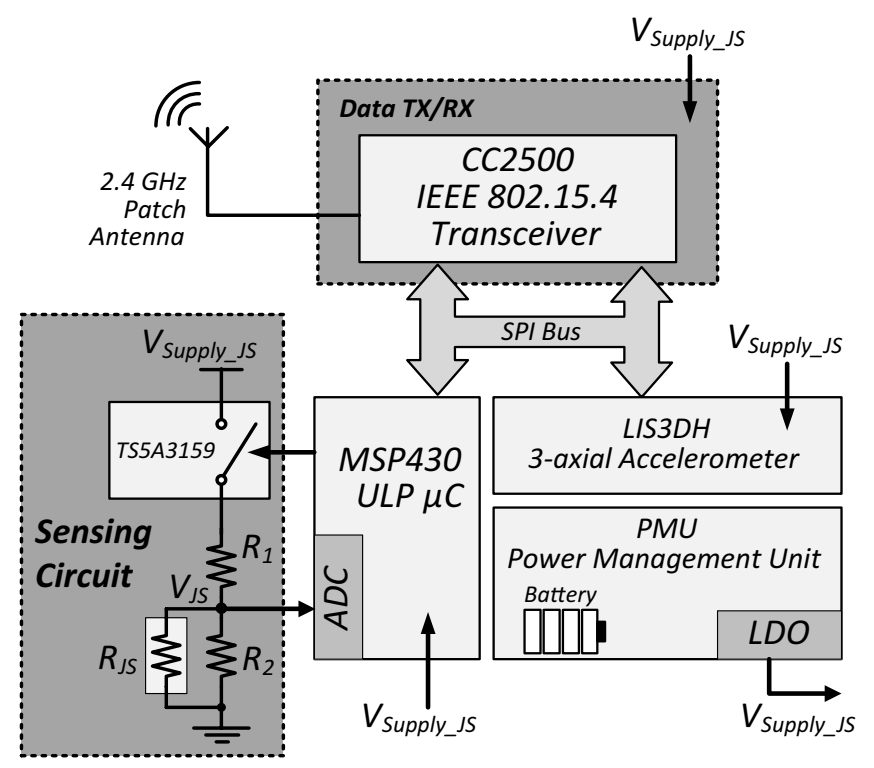

Fig. 7. Simplified Block Diagram of the Joint Sensor Device - JS.

easily controlled through a SPI communication by adding only few external components. This transceiver is intended for the usage in the $2.4 \mathrm{GHz}$ ISM (Industrial, Scientific and Medical) and SRD (Short Range Device) frequency band, being IEEE 802.15.4 compliant. Its data rate can be configured via software up to $500 \mathrm{kBaud}$, while typical current consumptions are $400 \mathrm{nA}$ when the transceiver is in power down mode, $21 \mathrm{~mA}$ when it is in transmit mode with an output power of $+1 \mathrm{dBm}$, and $13.3 \mathrm{~mA}$ when it is in receive mode.

The wear level of the joint is measured by means of a very simple circuitry. The basic principle is based on the classic voltage divider where the output voltage is the voltage drop across the joint, $V_{\mathrm{JS}}$ that varies over time during the normal joint operation from several mega ohms (new joint) to few ohms (worn out joint). This voltage is a fraction of the input voltage of the divider (i.e. the supply voltage of the sensor node, $\left.V_{\text {Supply_Js }}\right)$ and is proportional to the impedance measured on to the ball of the joint (i.e. between the upper bearing, part (2) in Figure 2, and the upper cup, part (5) in Fig. 2) accordingly with Equation (1):

$$
V_{\mathrm{JS}}=V_{\text {Supply_JS }} \frac{\left(R_{2} / / R_{\mathrm{JS}}\right)}{R_{1}+\left(R_{2} / / R_{\mathrm{JS}}\right)}
$$


where $R_{1}$ and $R_{2}$ are the voltage divider resistors and $R_{J S}$ is the resistance of the joint sensor indicating the wear level. The ON-resistance of the solid state switch is around $1 \mathrm{ohm}$, while $R_{1}$ and $R_{2}$ have to be chosen in the order of ten-hundreds of kilo-ohm in order to minimize the node power consumption. Consequently the voltage drop across the solid state switch has been neglected in Equation (1).

The $\mu \mathrm{C}$ periodically drives an ultra-low power solid state switch from Texas Instruments, i.e. TS5A3159, which enables the sensing circuitry. When the switch is closed the sensed impedance value, $R_{J S}$ is acquired through the Analog-to-Digital Converter (ADC) embedded in the $\mu \mathrm{C}$ and it is compared with a predefined wear threshold determined by means of experimental characterization on the joints degradation dynamics. If the impedance is higher than the predefined threshold the joint is in good conditions (and it can safely work), whereas if the impedance is lower than the predefined threshold the joint has to be replaced. In order to minimize the power consumption, the switch on the sensing circuitry has been designed to be normally open (see Fig. 7), thus allowing to limit the current consumption to the sensing interval i.e. when the solid state switch is closed

The JS is powered with a $450 \mathrm{mAh}$ rechargeable LiPoly battery. It includes a smart Power Management Unit (PMU), which exploits ultra-low power Linear-Dropout regulator (LDO), i.e. the TPS780270200 from Texas Instruments. This LDO has a surprisingly low quiescent current of $\approx 500 \mathrm{nA}$, and efficiency higher than $85 \%$ over the whole output current range. This unit is controlled by the $\mu \mathrm{C}$ enabling advanced power management monitoring and control.

The JS has been developed by exploiting a hardwaresoftware co-design approach aiming at minimizing the power consumption of both hardware components and the software running on the microcontroller. In fact, the only use of ultra-low power architectures does not automatically allow minimizing the power consumption of the system [39] and a smart use of the resources of the $\mu \mathrm{C}$ is mandatory to achieve this task.

The software architecture has been devised to allow the JS to exploit an ad hoc-task management algorithm which plays a key role to reduce the power consumption. The developed task manager defines 2 working states: (i) the sleep state, where the joint is assumed to be off duty; and (ii) the run state, where the joint is assumed to be on duty. The transition between the two states occurs if the 3 -axial accelerometer detects acceleration larger than $50 \mathrm{mg}$ on at least one of the three axes.

In sleep state we consider that the off-highway vehicle is not in use. The accelerometer is not expected to measure any appreciable acceleration until the vehicle will be in use. Consequently the microcontroller and the RF transceiver are kept in sleep mode in order to minimize power consumption. The only task executed in this state consists of waking up the system at regular intervals to check for vibration. When the JS detects acceleration the

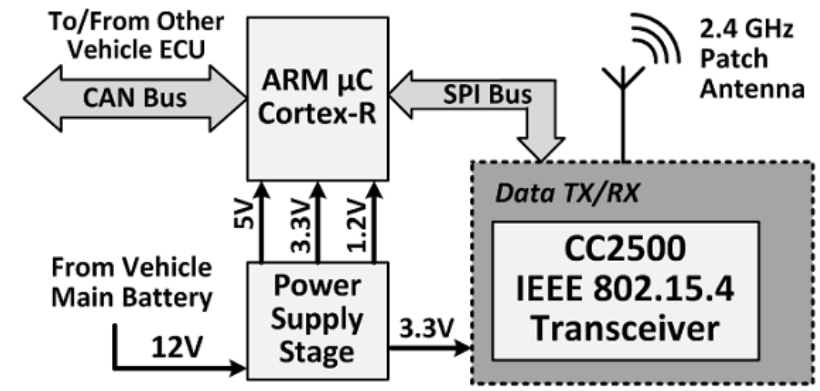

Fig. 8. Simplified Block Diagram of the Data Manager Device - DM.

task manager immediately switches from the sleep state to the run state.

In run state the JS sends periodically a data packet containing a unique identifier of the JS, a packet number, the estimated wear condition of the joint, the measured accelerations and a checksum field ensuring a minimum level of consistency of the wirelessly transmitted data. The checksum has been implemented in the classic hexadecimal format and it is calculated by a XOR of all characters in the message between two special characters used as delimiters of the sensible data fields. The checksum is calculated by the JS which transmits the data and by the Data Manager (DM) which receives the data. The DM checks if there is an agreement between the calculated checksum and the one received from the JS. In case of agreement the received data are unpacked, vice versa, the received message is discarded.

Interestingly, other wireless sensors can be easily realized exploiting the JS hardware platform, allowing monitoring other physical parameters with minimal software overload, mainly related to differences in the conditioning of the signals provided by different transducers

\section{Data manager}

Figure 8 shows the simplified block diagram of the implemented DM system. The core of the DM installed on the vehicle is an ARM Cortex-R4F processor, i.e. the RM48L952 microcontroller developed for systems from Texas Instruments. The main features of its safety architecture are a dual CPU operating in lockstep, a memory with built-in self-test logic for single bit error correction and double bit error detection, and a multiple inter-chip serial interfaces (i.e. UART, SPI, $\mathrm{I}^{2} \mathrm{C}$ and embedded CAN controllers). The DM works as communication bridge between JS and other ECUs installed on the vehicle (e.g. displays). The wireless communication between DM and JS devices is implemented by means of the same $2.4 \mathrm{GHz}$ transceiver used in the JS, i.e. the CC2500 transceiver controlled using a dedicated SPI bus. Of course DM and JS devices share the same communication protocol.

On the other hand, the communication between DM and other ECUs installed on the vehicle exploits a $250 \mathrm{Kbps}$ CAN communication bus. The DM device is 
connected to the bus through a SN65HVDA541 CAN transceiver from Texas Instruments qualified for use in automotive applications.

Differently from the JS node, the DM device has no power consumption constraints because it is powered by the main battery of the vehicle. Therefore all the voltages needed for the proper internal operation of the device blocks have to be generated by its power management starting from the $12 \mathrm{~V}$ DC of the vehicle battery. Commercial DC-DC converters from Texas Instruments are used to obtain a regulated voltage of $1.2 \mathrm{~V}$, which is required by the microcontroller core, a regulated voltage of $3.3 \mathrm{~V}$, which is used by microcontroller peripherals and CC2500 transceiver and a regulated voltage of $5 \mathrm{~V}$ used by the CAN transceiver

At this stage the DM has to perform tasks having a very low computational complexity hence its microcontroller is not used at its full potential Nevertheless, it has been chosen because of its internal architecture, which is specifically designed for safety critical applications (i.e. its redundant architecture with dual CPU in lock-step) Furthermore, it allows implementing of complex functions (e.g. working time counter, time to maintenance, graphical display of all sensible parameters coming from all the sensors on the vehicle) without hardware modifications.

\section{Experimental assessment}

The performances of the ball-joint wear sensor were assessed by testing the prototypes under real working conditions. Two were the aims of the experimental tests, each corresponding to a step of the work: (i) to choose the most appropriate coating for the wear sensor; (ii) to assess the system as a whole (instrumented ball joint and electronic system).

As described in the following, experimental tests on the coating have been carried out in laboratory using an ad-hoc test bench, while the tests of the whole system have been carried out installing two JS on a real tractor (one sensor on the left side of the front axle and one sensor on the right side) and monitoring the wear of the two JS in real working conditions.

\subsection{Assessment of the wear monitoring ball joint}

The experimental assessment was performed on the prototype of the ball joint implementing the wear sensor (Fig. 6). The experimental campaign examined each of the three types of coatings of the upper bearing described above (Rilsan, Loctite Nordbak 7227, and anodic oxidation on an aluminum bearing). In order to speed up the wearing of the ball joint, thus shortening the tests, only a minimum amount of lubricant was introduced inside the prototype and the polyurethane sleeve was removed. This condition emulates the damage of the polyurethane sleeve on the field, where only a small amount of Molykote stays inside the joint. The normal lubrication condition was not examined, since, according to previous tests performed by

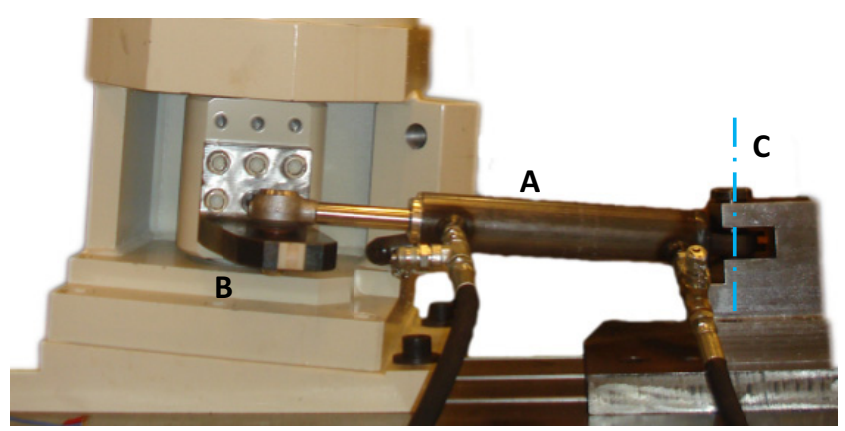

Fig. 9. Testing equipment for the ball joint wear sensor.

the industrial Partner, until the joint is properly sealed and lubricated, failure is completely prevented.

The sensor was monitored through a data acquisition module (USB 6251 from National Instruments [40]) which measured the electric resistance and registered the number of cycles up to failure. The failure of the system was assumed to occur when the impedance between the upper bearing and the external frame of the ball joint dropped down to $50 \%$ of the initial value. Three replicates were performed for each type of coating, and the ball joint was tested up to failure.

The test procedure aims to reproduce the fatigue loading which affects the ball joint in real working conditions. According to the proprietary standard from Ognibene, the test bench has to mimic the same geometry and kinematics of the front axle of a tractor, in order to describe the typical motion law occurring in service conditions. The cycles are performed at a frequency equal to $0.2 \mathrm{~Hz}$, by controlling the force. The load on the joint equals $70 \%$ of the maximum shear force for the ball joint along the stroke, while reaches $100 \%$ of the maximum shear force at the end of stroke. The tests were performed through the ad-hoc hydraulic testing machine available in the Ognibene research lab (Fig. 9). This test bench reproduces on the ball joint the motion law and forces prescribed by the company standard, which was inspired by the Japanese automotive standard (JASO-C615-89) [39]. Thanks to a peculiar kinematic link, the ball-ended pin axis sweeps a conical surface during each cycle, with an opening angle of $20^{\circ}$. This motion law is obtained by combining the alternate translation of the piston rod ( $\mathrm{A}$ in Fig. 9) which rotates on the hinge $\mathrm{C}$, to the cyclic rotation of the bracket connected to the hydraulic brake simulating the wheel spindle (B, in Fig. 9), inclined with respect to the hinge $\mathrm{C}$. The test machine is equipped with a $25 \mathrm{kN}$ load cell installed along the axis of the piston and is supplied by an $11 \mathrm{~kW}$ hydraulic power unit. The whole system is controlled through a hardware in the loop program developed in Labview [40].

Figure 10 shows in detail the combination of the motion law and the loading cycle applied to the system in the experimental tests, which originate a maximum shear force on the ball- ended pin of the joint up to about $16 \mathrm{kN}$. 


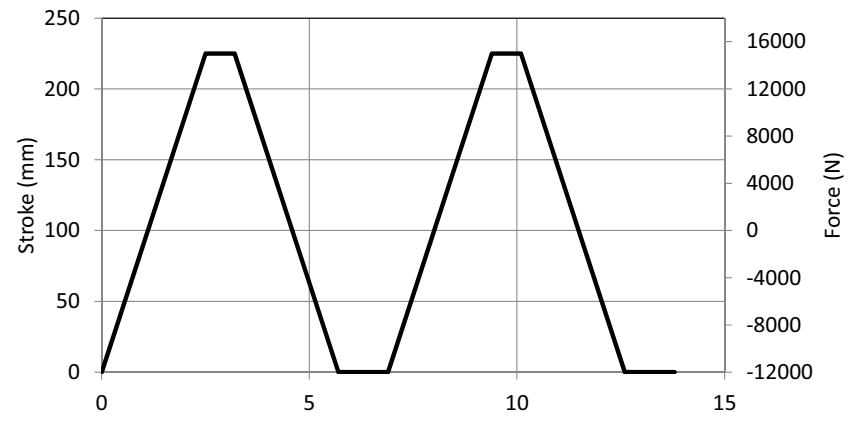

Fig. 10. Motion law on the steering actuator during the test.

\subsection{Assessment of the mechatronic system prototype}

A Landini Power Mondial 120 tractor [41] was used for the validation of the prototype in real working scenarios. Figure 11 shows a prototype of the ball joint including the JS enclosed in the rapid prototyped white ABS box. On each connection of the front axle of the tractor with the wheel spindles, we installed a prototype, including an aluminum bearing with anodic oxidation. Both wear sensors were managed by the DM device installed in the cabin.

In order to assess the system response, the endurance test was performed in a test track at Ognibene site, including rough terrain conditions. To speed up the test, both ball joints were assembled without Molikote lubricating grease, and the test lasts until worn out of both joints occurred.

The DM collected the data received from the Joint Sensors. In normal working conditions, the collected data are forwarded via CAN bus to the other vehicle ECUs, but for the specific test presented in this paper the firmware of the DM has been slightly modified in order to forward the collected data also through a classic serial UART communication (9600bps, 8-N-1). By connecting a laptop to the DM, it has been possible to sniff the collected data and visualize the associated information by means of a terminal emulator.

The JS awakes periodically (30 s of sampling time) from its ultra-low power consumption mode to check for vibrations and measuring the correspondent accelerations along each of its three physical axes. If at least one component results higher than a defined threshold (i.e. $50 \mathrm{mg}$ ), the JS is considered on duty, the sensing circuitry is enabled, the wear level of the joint (i.e. the joint impedance) is measured, and the relative estimated condition is sent wirelessly to the DM together with the measured $x_{-}, y_{-}$, $z$-accelerations.

\section{Results and discussion}

\subsection{Assessment of the wear monitoring ball joint}

Table 4 collects the average number of cycles up to failure for each type of coating. At the end of each test, the ball joint was disassembled and the coated bearings

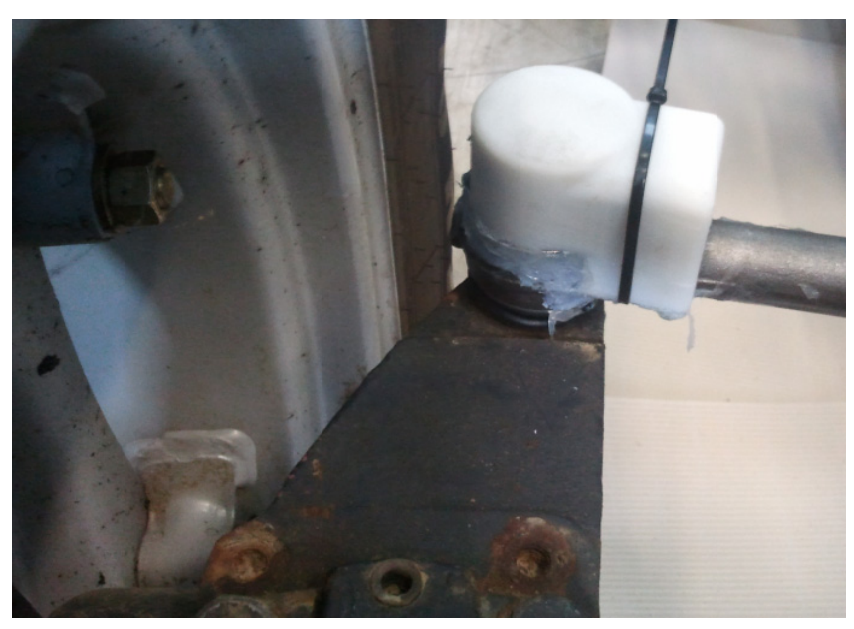

Fig. 11. Prototype of the ball joint including the wear sensor and the electronic board encapsulated in a plastic case, installed on the test tractor.

Table 4. Results from the experimental tests.

\begin{tabular}{cc}
\hline Coating type & $\begin{array}{c}\text { Average number of } \\
\text { cycles before failure }\end{array}$ \\
\hline Rilsan & 22925 \\
Loctite Nordbak 7227 & 14321 \\
Anodic oxidation & 35260 \\
\hline
\end{tabular}

carefully examined (Fig. 12). From this visual examination it appeared that the lack of lubricant had produced damaging of coating and hence a drop down of electric resistance occurred.

With regard to the different types of coatings, all of them provide a good wear resistance before failure, but with regard to manufacturing, the Rilsan coating and the anodic oxidation are easier to be produced.

Since the tests were performed according to the Ognibene company procedure, even though they are compatible with the Japanese standard [39], they are specific for agricultural equipment, the core business of the company, and it is not possible to compare these results against data available from the literature. However, the main aim of these tests is to compare the wear resistance of the three different coatings here examined, in order to identify the more durable and reliable.

\subsection{Assessment of the mechatronic system prototype}

The terminal emulator used to visualize the data collected by the DM gives many information. The first message informs about the DM status and its proper operation. If within 30 seconds no sensors send their data packet the DM notifies that there are no JS or other kind of sensors installed on the vehicle on duty (second message). Vice versa when the installed Joint sensors are on duty the DM processes the received data and forwards them via UART.

The data packet transmitted on the UART is comprised of (in order of appearance): an header, the unique 


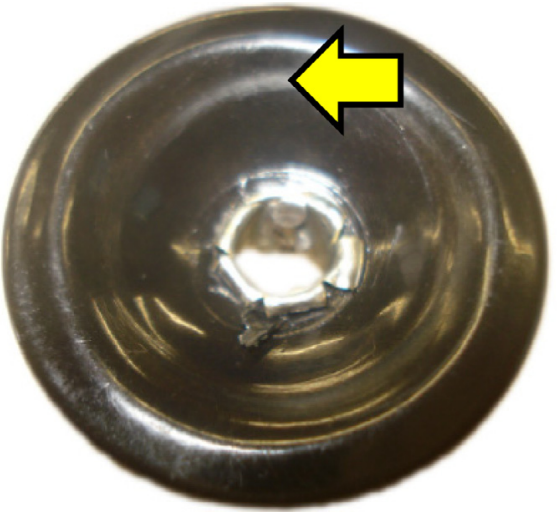

(a)

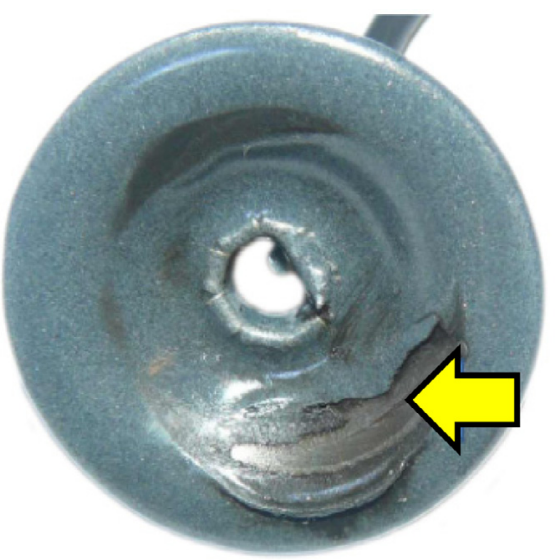

(b)

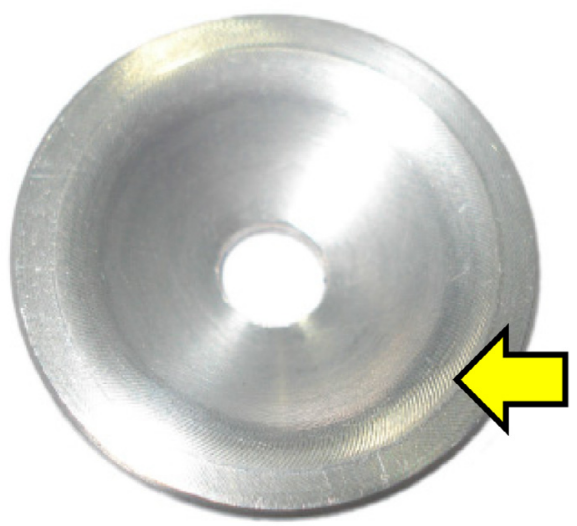

(c)

Fig. 12. Failed upper bearing coated with Rilsan (a), Loctite Nordbak 7227 (b), and anodized aluminum (c).

identifier of the JS, the packet number, the measured $x$ $y$ - and $z$-components of the accelerations (expressed in $\mathrm{mg}$ ) and the estimated wear condition of the joint. Only data packet received correctly is displayed on the terminal emulator. The checksum calculated by the DM to verify the consistency of the wirelessly received data packet is not included in the UART data packet.

Once the JS device detects an excessive wear of the joint where it is installed, the failure is notified by the change in the last field of the UART data packet.
Field tests showed that both ball joints installed on the front axis wheel without lubricating grease gave a failure message to the managing software. Disassembly of the joints highlighted that this message, as expected, occurred due to worn out of the insulating alumina layer on the upper conical bearing.

The validation test on the prototype proves that this concept of wear sensor is a simple and reliable system to monitor the wear of the ball joint. Thanks to its ultra-low power consumption ( $\approx 5 \mu \mathrm{A}$ in sleep mode), the JS can be made autonomous through an energy harvesting device, able to gather energy from the natural vibrations of the front axle occurring when the machine is on duty.

\section{Conclusions}

The work presented the development and validation of an innovative ball joint wear sensor for monitoring and diagnosis of off-highway vehicles. This kind of sensor is not currently available in the market and has very innovative features like: ultra-low power consumption that enables self-powering through energy harvesting, wireless connectivity, small size, low-cost and easy integration in existing ball joints. The ball joint wear sensor enables an active monitoring of wear and damaging, thus enhancing safety, reliability and efficiency of off-highway vehicles. In fact, the sensor was developed to monitor the wear in a safety-critical ball joint, which connect the steering actuator and the wheel spindle, but is potentially applicable to many kind of ball joints. The work presented the conceptual design and development both of the mechanical and electronic system for sensor management. The assessment of a sensor prototype on an agricultural tractor proved that the sensor is a simple and reliable system for wear monitoring, able to diagnose incoming failure that would cause a complete loss of the steering action. The ultralow power consumption of the joint sensor make possible to develop an autonomous system by including an energy harvesting device able to gather energy from the natural vibration occurring on the joint when on duty.

Acknowledgements. Emilia Romagna Region is gratefully acknowledged for founding the Isotractor project and Ognibene Power S.p.A. for its kind cooperation.

\section{References}

[1] http://www.moogproblemsolver.com/_pdf_en2/DYK09_ 101_En.pdf

[2] M. Shuster, G. Maughan, R. Arnold, Development of a Maintenance Free Self-Lubricating Ball Joint, SAE Technical Paper, 1999, 1999-01-0036

[3] A.E. Samuel, The Winning Line: A Forensic Engineer's Casebook, Springer, 2007

[4] E.A. Ossa, C.C. Palacio, M.A. Paniagua, Failure analysis of a car suspension system ball joint, Engineering Failure Analysis 18 (2011) 1388-1394 
[5] K. Baynal, M. Makaraci, K. Gulbudak, Solution for failure analysis of automotive axle knuckle pull-out, Int. J. Automotive Technol. 11 (2010) 701-710

[6] B.H. Jang, K.H. Lee, Analysis and design of a ball joint, considering manufacturing process, Proc IMechE Part C: J. Mech. Eng. Sci. 18 (2013) 146-151

[7] H. Hirabayashi, K. Terai, A. Matsushima, A study of dust cover seals for ball joint application, SAE Technical Paper 1977770176

[8] H. Martins, M. Zucchini, Ball joint pull out simulation using finite element analysis, SAE Technical Paper, 2006 2006-01-2867

[9] N. Durisek, K. Granat, E. Holmes, Analysis of Front Suspension Ball Joint Separations in Motor Vehicle Crashes, SAE Technical Paper 2009 2009-01-0101

[10] S.R. Goodrich, J.M. Roethlisberger, Ball joint with manually operable, sensible wear indicator. Patent No. 3791748, 1974

[11] T. Tajima, T. Tsuji, Pivot joint assembly capable of amplified wear indication, Patent No. 4626121, 1986

[12] G.C. Parker, Device and method for closing a movable socket and establishing a predetermined wear indicator distance. Patent No. 6125541, 2000

[13] D.E. Williams, Ball joint assembly with wear indication. Patent No. 7048461, 2006

[14] C.D. Benick, P.E. Jacobson, Ball joint assembly with wear indicating electrical circuit. Patent 2006/0029461 A1, 2006

[15] K. Ulrich, S. Eppinger, Product Design and Development, 5th edn. McGraw-Hill, New York, 2011

[16] http://www.elettronica.unimore.it/site/home/ technology-transfer/isotractor.html

[17] F. Sarghini, G. D'Urso, An Early Warning Device for Identification of Tractor Accidents, Rapid Alert and Assistance, SHWA2010 International Conference on Work Safety and Risk Prevention in Agro-food and Forest Systems, September 16-18, 2010 Ragusa, Italy, pp. 494500

[18] C. Casazza, V. Rondelli, R. Martelli, A critical evaluation of data collection on tractor rollover accidents in Italy, Proceedings International Conference of Agricultural Engineering, 6-10 July 2014, Zurich, 2014

[19] European Road Safety Charter, http://www. erscharter.eu/en

[20] J.R. Myers, K.J. Hendricks, Agricultural tractor overturn deaths: assessment of trends and risk factors, Am. J. Ind. Med . 53 (2009) 662-672

[21] National Agricultural Statistics Service. 2006 farm and ranch safety survey. Washington, DC: U.S. Department of Agriculture, National Agricultural Statistics Service, 2008, Report No. Sp Cr 3-1 (1-08)
[22] Committee on Agricultural Safety and Health Research and Extension. 2009. Agricultural Equipment on Public Roads. USDA-CSREES, Washington, DC

[23] B. Mark, M. Hobelsberger, Switch for a brush wear recording circuit. Patent n. US 2009/0266700 A1, 2009

[24] T.A. Brey, Tire tread wear sensor system. Patent No. 7180409,2007

[25] C. Benick, P. Jacobson, Ball joint assembly with wear indicating electrical circuit. Patent No. US 2006/0029461 A1, 2006

[26] A. Ciotti Device for indicating caliper pad wear in disc brakes. Patent No. 6272914, 2001

[27] D. Pullini Tire wear monitoring system. Patent No. 7353700, 2008

[28] D.R. Gaines, F.J. Bronco, Wear indicator for ball joints. Patent No. 3960457, 1976

[29] K.F. Dresselhouse Ball joint having service life indicator. Patent No. 5163769, 1992

[30] E. Gunter, Method for monitoring mechanical wear. Patent No. 6868711, 2005

[31] M. Eguchi, Motor having a bearing wear monitoring device, Patent No. 6114966, 2000

[32] G. Flores, H. Reisser H, Lex K. Honing or grinding tool and measuring device for measuring wear. Patent No. 5144773, 1992

[33] R.H. Hammerle, Resistive oil quality sensor. Patent No. 5332961, 1994

[34] H. Kwun, A.S. Birring, G.P. Singh, G.J. Hendrix D.G. Alcazar DG Method of wear monitoring using ultrasonic phase comparison. Patent No. 4711120, 1987

[35] J.D. White, J.R. Clark, L.A. Caron, Vehicle friction material condition measurement system. Patent No. 5559286, 1996

[36] J.D. Hedges, P.J. Voelker, Oil monitoring system. Patent No. 7928741, 2011

[37] J.M. Fildes, R.H. Krueger, Cutting tool wear monitor. Patent No. 4694686, 1987

[38] http://www.loctite-kleje.pl/sg_obrazki_/ 00002363_zalac_003.pdf

[39] Japanese Automobile Standard, Bench Test Procedures of Suspension Ball Joint for Independent Type, JASO C615-89, 1989

[40] http://www.ni.com/products/

[41] http://www.argotractors.com/it/

[42] A. Bertacchini, G. Napoletano, D. Dondi, L. Larcher, P. Pavan, Optimized Energy-Aware Wireless System for Identification of the Relative Positioning of Articulated Systems in the Free Space Sensors Journal IEEE 14 (2014) 1682-1692 